\title{
Energetics and Possible formation and decay mechanisms of Vortices in Helium Nanodroplets
}

\author{
Kevin K. Lehmann* and Roman Schmied \\ Department of Chemistry, Princeton University, Princeton, New Jersey 08544
}

(Dated: June 9, 2018)

\begin{abstract}
The energy and angular momentum of both straight and curved vortex states of a helium nanodroplet are examined as a function of droplet size. For droplets in the size range of many experiments, it is found that during the pickup of heavy solutes, a significant fraction of events deposit sufficient energy and angular momentum to form a straight vortex line. Curved vortex lines exist down to nearly zero angular momentum and energy, and thus could in principle form in almost any collision. Further, the coalescence of smaller droplets during the cooling by expansion could also deposit sufficient angular momentum to form vortex lines. Despite their high energy, most vortices are predicted to be stable at the final temperature $(0.38 \mathrm{~K})$ of helium nanodroplets due to lack of decay channels that conserve both energy and angular momentum.
\end{abstract}

Vortices are an almost unavoidable presence in bulk superfluid helium. There is a rich history of studies of their properties and interactions $\underline{\underline{1}}$ It is natural to consider their possible presence in the finite superfluid found in ${ }^{4} \mathrm{He}$ nanodroplets $\stackrel{2}{=}$ The study of helium nanodroplets has been quite active in recent years, starting with the spectroscopy of embedded molecules as a probe for properties of nanodroplets, which was introduced by Scoles and coworkers ${ }^{3}$ However, despite a now large body of work, no unambiguous signature of the presence of vortices has yet been reported. This is perhaps surprising, as a vortex in a nanodroplet is expected to bind a molecular impurity ${ }^{4.5}$ and likely introduce a highly anisotropic interaction potential, though no explicit calculation of the magnitude of such an anisotropy has been reported to date. The vortex-induced anisotropy in molecular orientation, if large compared to the rotational constant of the molecule in liquid helium, will quench the molecular rotation and collapse the rotational structure that is one of the hallmarks of molecular spectroscopy in helium nanodroplets $\frac{6.7}{.7}$

Several calculations have been published for the energy of a droplet with a straight vortex, with and without an atom or cylindrically symmetric molecule aligned with the vortex ${ }^{4.5,8.9}$ As in bulk superfluid, these calculations have found the energy of droplets with a straight vortex to be significantly higher than that of vortex-free droplets $\frac{4.5}{4}$ This result, combined with the failure to observe vortex lines to date, have led some to propose that vortices are unstable in helium nanodroplets and perhaps are rapidly expelled. In this paper, we revisit the energetics of vortex lines, considering both the straight vortex line down the center of the droplet that has previously been considered and curved vortex lines that will rotate around the droplet due to their own flow field. Furthermore, we consider the effect of angular momentum conservation on the possible formation and decay of vortices in droplets. It turns out that the linear and curved vortices contain considerably less excitation energy per unit angular momentum than the final states accessible by decay through the mechanisms considered. This suggests that despite their higher energy than the ground state, droplets with such vortices are the lowest possible states with high angular momentum, and thus should in fact be stable to decay.

\section{HOLLOW CORE MODEL OF VORTEX LINES}

There have been several microscopic calculations of the properties of straight vortex lines in helium nanodroplets, both pure and doped with atomic or molecular solutes 4.5 .8 .9 .10 .11 In this work, we exploit the phenomenological description known as the hollow core model. The numerous microscopic treatments of vortex lines in two and three dimensions have largely confirmed its qualitative applicability 12.13 .14 .15 .16 .17 and it is widely used to describe the properties of vortex lines and rings in bulk liquid helium.1.18

The vortex is surrounded by a circulating flow, characterized by an irrotational velocity field $(\boldsymbol{\nabla} \times \boldsymbol{v}=0)$. In the simple case of a straight vortex in bulk helium, the magnitude $v$ of the flow velocity at any given point is inversely proportional to the distance of that point from the center of the vortex, $v=\hbar / m r$ ( $m$ is the mass of a ${ }^{4} \mathrm{He}$ atom). This leads to a kinetic energy density whose volume integral diverges as the vortex is approached $(r \rightarrow 0)$. In the hollow core model, the helium number density $\rho$ is taken to be zero inside a cylinder of radius $a$ and equal to the bulk value $\rho_{\mathrm{b}}=0.0218 \AA^{-3}$ outside of this cylinder. Such a discontinuous change in density is unphysical, but microcanonical calculations have confirmed a nearly hollow core, though with a smooth transition of the density to the bulk value 13.15 It is noted that our model treats the helium density on the outer boundary of the droplet as abruptly going to zero, while it is known that in fact the surface of liquid helium is diffuse, with a thickness of $\approx 6-8 \AA .19$ A hollow core radius of $\tilde{a}=1.00 \AA$ was found experimentally 18 to best reproduce the measured energy and velocity of vortex rings in bulk liquid helium; matching our expression of the velocity of vortex rings to the expression in Ref. 18 requires us to use $a=\tilde{a} / \sqrt{e}=0.607 \AA$ (see Appendix $\mathrm{A}$ ). Any normal fluid component is neglected as helium nan- 
odroplets in this size range have no thermally excited phonon excitations ${ }^{20}$

First we consider a straight vortex through the center of a helium nanodroplet. Because of the loss of spherical symmetry, we will consider that droplet to have the shape of an ellipsoid, with axial radius $B$ and equatorial radius $A$; the density is assumed to be uniform, equal to the bulk value $\rho_{\mathrm{b}}$, and dropping to zero at the ellipsoidal surface. In this geometry, the flow field is the same as for the bulk straight vortex described above. The kinetic energy, $E_{\mathrm{sv}}$, of this straight vortex flow field is given by

$$
\begin{aligned}
E_{\mathrm{sv}}= & \frac{h^{2} \rho_{\mathrm{b}} B}{2 \pi m}\left[\frac{1}{2} \ln \left(\frac{A+\sqrt{A^{2}-a^{2}}}{A-\sqrt{A^{2}-a^{2}}}\right)-\frac{\sqrt{A^{2}-a^{2}}}{A}\right] \\
& \rightarrow \frac{h^{2} \rho_{\mathrm{b}} B}{2 \pi m}\left[\ln \left(\frac{2 A}{a}\right)-1\right] \quad \text { for } A \gg a .
\end{aligned}
$$

For a spherical droplet of $N$ atoms, $B=A=R=$ $\sqrt{r_{0}^{2} N^{2 / 3}+a^{2}}$, with $r_{0}=\left(4 \pi \rho_{\mathrm{b}} / 3\right)^{-1 / 3}=2.22 \AA$.

An interesting question is the extent to which the large angular momentum of the vortex will distort the otherwise spherical droplet. Making the droplet oblate will reduce the length of the vortex line and thus lower its energy. However, this will also increase the surface energy of the droplet. The latter is the product of the surface tension of bulk liquid helium, $\sigma=0.272 \mathrm{~K}^{-2}, 21$ and the surface area of an ellipsoid, $S=2 \pi A^{2}+\frac{\pi B^{2}}{e} \ln \left(\frac{1+e}{1-e}\right)$, with the eccentricity $e=\sqrt{1-(B / A)^{2}}$. The droplet distortion is found by minimizing the sum of the vortex kinetic energy (given by Eq. (II)) and the surface energy of the droplet, at constant droplet volume $V=$ $\frac{4 \pi}{3} \frac{B}{A}\left(A^{2}-a^{2}\right)^{3 / 2}$. Table】gives the resulting eccentricity, $e$, and the associated stabilization energy (reduction in energy from a spherical droplet of the same volume with vortex). It is seen that the distortion from spherical symmetry is small, in the sense that $B / A=\sqrt{1-e^{2}}$ is close to 1 , despite the high angular momentum of the vortex, and this distortion will be neglected in the rest of this paper.

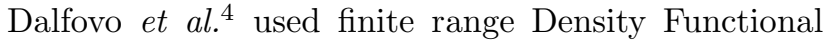
Theory (DFT) to calculate the energy and core shape of a straight line vortex for a range of droplet sizes $(N=$ $50-1000)$. While their core has a smooth density profile, they report a core radius of the order of $1-2 \AA$. Figure 1 shows a plot of the vortex energy calculated by the hollow core model as a function of the number of helium atoms in the droplet, along with the same quantity estimated by the Density Functional ${ }^{4}$ method. The hollow core model appears to slightly underestimate the vortex energy with respect to DFT; agreement with the DFT calculation can be made almost quantitative if a value of $a=0.56 \AA$ is used. The core radius used in the hollow core model is to be interpreted as an effective radius that reproduces the energetics and velocity of experimental vortices, and not more than an estimate of the real core radius.

The angular momentum associated with a straight vortex is $N \hbar$, i.e., one unit per helium atom. Both methods

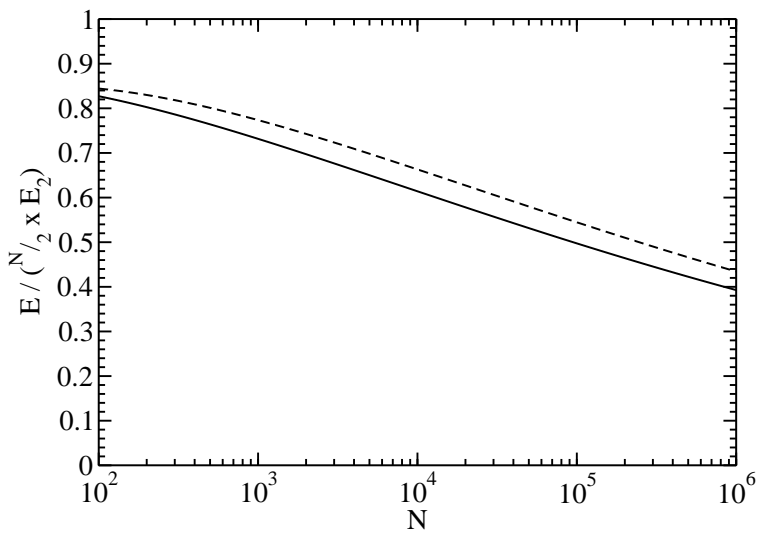

FIG. 1: Energy of the straight vortex line solution as a function of the number of $\mathrm{He}$ atoms in the droplet, calculated both by the Hollow Core model (solid line, Eq. (1)) and by finite range Density Functional Theory (dashed line, Ref. 4). Energies are displayed as a fraction of the minimum energy required to deposit $N \hbar$ units of angular momentum into ripplon excitation modes.

agree that the vortex energy per unit angular momentum is lower than for any other excitation mode of a pure helium droplet: ${ }^{20}$ Figure 1 shows the vortex excitation energies relative to the energy required to produce $N / 2$ quanta of $L=2$ ripplons, which is the lowest energy state based upon quasiparticle excitations that has the same total angular momentum as the straight vortex. Table shows a comparison of the hollow core model excitation energy of a straight vortex and this lowest ripplon, and their energies per unit angular momentum for droplets of the size range that span most helium nanodroplet experiments. As will be discussed below, it is the relatively low energy per unit angular momentum that is key to the proposed metastability of vortex line solutions.

\section{CURVED VORTEX LINE SOLUTIONS}

We now turn to more general vortex line solutions. The flow field around a general vortex line in bulk helium is homologous to that of the magnetic field around a curved wire that follows the vortex line. The "current" in this wire is proportional to the quantum of circulation, $\kappa=$ $h / m$. Thus the flow field $\boldsymbol{v}(\boldsymbol{r})$ is given by the Biot-Savart equation ${ }^{22}$

$$
\boldsymbol{v}(\boldsymbol{r})=\frac{\kappa}{4 \pi} \int_{\text {vortex }} \frac{(\boldsymbol{s}-\boldsymbol{r}) \times \mathrm{d} \boldsymbol{s}}{|\boldsymbol{s}-\boldsymbol{r}|^{3}} .
$$

The vortex must either form a closed loop or end at a boundary of the superfluid helium. $\boldsymbol{v}(\boldsymbol{r})$ must not have a normal component at any boundary of the superfluid. This implies that the vortex must intersect a helium boundary at normal incidence.

A curved vortex will move in its own flow field. When the local radius of curvature of the vortex $\mathcal{R}(\boldsymbol{s})$ is large 
TABLE I: Properties of Helium droplets and their straight vortex solutions as a function of the number of Helium atoms.

\begin{tabular}{lcccccl}
\hline \hline He Number & $10^{2}$ & $10^{3}$ & $10^{4}$ & $10^{5}$ & $10^{6}$ & \\
\hline Radius $R$ & 1.033 & 2.222 & 4.785 & 10.31 & 22.21 & $\mathrm{~nm}$ \\
Helium Binding Energy & 4.72 & 6.03 & 6.63 & 6.91 & 7.04 & $\mathrm{~K}$ \\
Total Thermal Ripplon Energy & 0.413 & 3.56 & 18.5 & 87.0 & 403. & $\mathrm{~K}$ \\
$L=2$ Ripplon Energy & 1.05 & 0.332 & 0.105 & 0.0332 & 0.0105 & $\mathrm{~K}$ \\
Thermal Ripplon $\sqrt{\langle L(L+1)\rangle}$ & 1.54 & 8.54 & 38.7 & 173. & 781. & $\hbar$ \\
Vortex Energy $E_{\mathrm{DFT}}$ & 44.3 & 129. & 349. & 905. & 2283. & $\mathrm{~K}$ \\
Vortex Energy $E_{\mathrm{HC}}($ Hollow Core) & 43.4 & 122. & 323. & 827. & 2064. & $\mathrm{~K}$ \\
Vortex Angular Momentum $L_{\mathrm{v}}$ & $10^{2}$ & $10^{3}$ & $10^{4}$ & $10^{5}$ & $10^{6}$ & $\hbar$ \\
$E_{\mathrm{HC}} / L_{\mathrm{v}}$ & 56.8 & 15.9 & 4.23 & 1.08 & 0.270 & $\mathrm{GHz}$ \\
Lowest Ripplon $E / L$ & 68.6 & 21.8 & 6.88 & 2.18 & 0.688 & $\mathrm{GHz}$ \\
\hline Vortex-induced eccentricity & 0.53 & 0.44 & 0.35 & 0.27 & 0.20 & \\
B/A & 0.85 & 0.90 & 0.94 & 0.96 & 0.98 & \\
Deformation Stabilization Energy & 2.00 & 3.74 & 6.16 & 9.22 & 12.9 & $\mathrm{~K}$ \\
\hline Max. $L$ loss by 1 atom evaporation & 26 & 96 & 345 & 1198 & 4090 & $\hbar$ \\
Max. $L$ loss by $n$ atom evaporation & 41 & 222 & 1213 & 6561 & 35000 & $\hbar$ \\
for evaporation of $n=$ & 5 & 10 & 25 & 59 & 146 & $\mathrm{atoms}$ \\
Max. $L$ loss by fission & 28 & 210 & 1488 & 10150 & 67400 & $\hbar$ \\
\hline$x_{0}$ for stability limit of curved vortex & 0.72 & 0.76 & 0.81 & 0.85 & 0.89 & $R(N)$ \\
Min. $L$ for stable vortex & 18 & 131 & 850 & $5.14 \times 10^{3}$ & $2.96 \times 10^{4}$ & $\hbar$ \\
Min. $E$ for stable vortex & 9.42 & 27.2 & 62.1 & 126. & 242. & $\mathrm{~K}$ \\
Max. $v$ for stable vortex & 56.3 & 39.0 & 27.6 & 19.5 & 13.8 & $\mathrm{~m} \mathrm{~s}{ }^{-1}$ \\
Max. kinetic energy of 100 u dopant & 19.1 & 9.1 & 4.6 & 2.3 & 1.1 & $\mathrm{~K}$ \\
\hline \hline
\end{tabular}

compared to its core radius $a$, the motion of the vortex core can be calculated using the Local Induction Approximation (see Appendix B and Ref. 18). The shape of the vortex core surface is determined by the condition of no helium flow across it, and turns out to be circular in cross section as long as $a \ll \mathcal{R}$. For finite core size, matching this boundary condition requires either that the core shape be altered or that one add an irrotational solution to Laplace's equation that corrects the normal component of velocity. It is not evident to the authors which change to make, and so these errors are neglected in the rest of the paper.

We now specialize to the case of a vortex line inside a spherical droplet of radius $R$. Muirhead, Vinen, and Donnelly ${ }^{23}$ showed that for any arbitrary vortex shape in a spherical droplet, the boundary conditions of the flow velocity on the surface of the droplet can be satisfied by continuing an image vortex outside the droplet. Each point on the vortex $s$ ( $s$ is the magnitude of this vector) generates an image point $s_{\mathrm{i}}=(R / s)^{2} s$ with vorticity equal to $-\kappa s / R$. Vorticity is conserved by attaching to each point along the image vortex a radially pointing vortex going to infinity that has a circulation strength given by the decrease in circulation of the image along its length. These all combine in the Biot-Savart equation to give

$$
\begin{array}{r}
\boldsymbol{v}(\boldsymbol{r})=\frac{\kappa}{4 \pi} \int_{\text {vortex }}\left(\frac{(\boldsymbol{s}-\boldsymbol{r}) \times \mathrm{d} \boldsymbol{s}}{|\boldsymbol{s}-\boldsymbol{r}|^{3}}-\frac{s}{R} \cdot \frac{\left(\boldsymbol{s}_{\mathrm{i}}-\boldsymbol{r}\right) \times \mathrm{d} \boldsymbol{s}_{\mathrm{i}}}{\left|\boldsymbol{s}_{\mathrm{i}}-\boldsymbol{r}\right|^{3}}\right. \\
\left.-\frac{\boldsymbol{s} \cdot \mathrm{d} \boldsymbol{s}}{R} \cdot \frac{\boldsymbol{r} \times \boldsymbol{s}}{s^{2}\left|\boldsymbol{s}_{\mathrm{i}}-\boldsymbol{r}\right|^{2}+s\left|\boldsymbol{s}_{\mathrm{i}}-\boldsymbol{r}\right|\left(R^{2}-\boldsymbol{r} \cdot \boldsymbol{s}\right)}\right),
\end{array}
$$

where

$$
\mathrm{d} s_{\mathrm{i}}=R^{2}\left(\frac{\mathrm{d} s}{s^{2}}-\frac{2(s \cdot \mathrm{d} s) s}{s^{4}}\right) .
$$

The three terms in the integral arise from the vortex, image vortex, and vorticity conserving radial vortex lines, respectively, and make contributions that decrease in magnitude the order given.

We seek curved vortex line solutions $\{x(\ell), z(\ell)\}$ that rotate at constant angular velocity $\Omega$ around the $z$ axis, which implies that these vortex line solutions will have constant shape. Appendix B gives the numerical procedure used to determine these solutions. Figure 2 shows some of the solutions for several values of $x_{0}$, the distance of minimum approach to the $z$ axis.

Bauer, Donnelly, and Vinen ${ }^{22}$ showed that the total angular momentum $\boldsymbol{L}_{\mathrm{v}}$ and kinetic energy $E_{\mathrm{v}}$ of the helium flow (which are defined by volume integrals for the corresponding densities) can be reduced to two surface integrals

$$
\begin{aligned}
& \boldsymbol{L}_{\mathrm{v}}=m \rho_{\mathrm{b}} \kappa \int \boldsymbol{r} \times \mathrm{d} \boldsymbol{S} \\
& E_{\mathrm{v}}=\frac{1}{2} m \rho_{\mathrm{b}} \kappa \int \boldsymbol{v} \cdot \mathrm{d} \boldsymbol{S},
\end{aligned}
$$

where the integration is over the the region in the $x z$ plane bounded by the vortex and the surface of the droplet. $\boldsymbol{v}$ in Eq. (6) is given by Eq. (31). The origin for vector $\boldsymbol{r}$ in Eq. (15) must be taken as the center of the sphere so that the outer surface of the sphere does not contribute to this integral expression. These expressions have neglected a contribution of the integral over 
the surface of the vortex core, but that should be small as long as $\mathcal{R} \gg a$ as required by our approximations. In the Appendix, we give explicit expressions for the lowest order (in $a / \mathcal{R}$ ) core surface corrections to $L_{\mathrm{v}}$ and $E_{\mathrm{v}}$, which are the calculations reported below. For the vortices considered here, $\boldsymbol{L}_{\mathrm{v}}$ is parallel to the $z$ axis owing to the symmetry of the vortex and its flow field with respect to reflection in the $x y$ plane. Calculation of $E_{\mathrm{v}}$ requires evaluation of a triple integral, with an integrand that is nearly singular along one of the edges of the integration domain; one of the integrations can be done analytically, leaving a double numerical integration. In the same way, the double integral for $L_{\mathrm{v}}$ can be reduced to a single numerical integration.

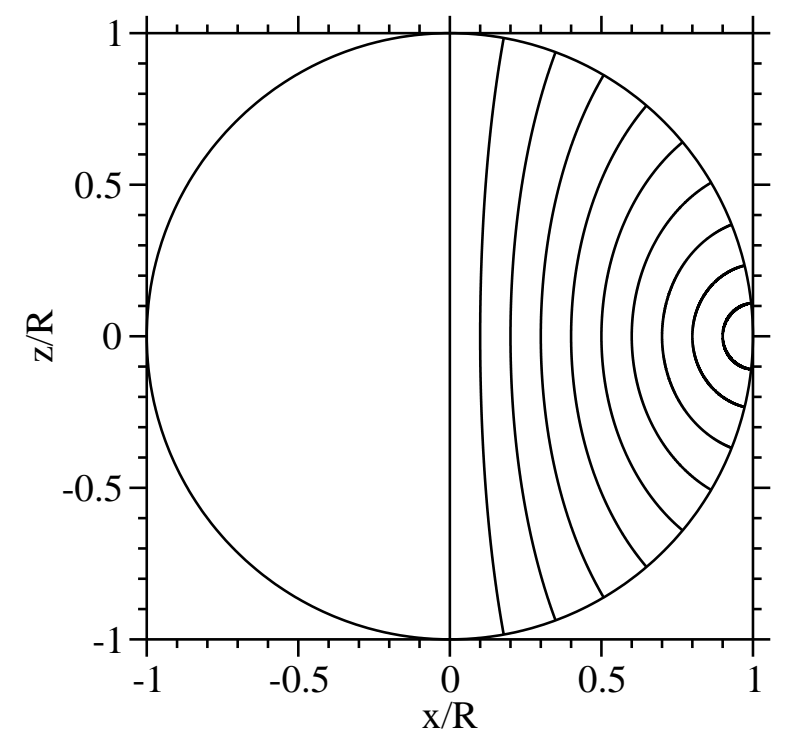

FIG. 2: Curved vortex solutions for different values of $x_{0}$, the distance of closest approach to the $z$ axis, in a droplet of $N=10000$ He atoms.

The treatment as yet is for vortex lines as classical objects with angular momentum pointing in a definite direction in space. By the standard rules of semiclassical quantization, vortex eigenstates can be constructed as linear combinations of the vortex lines with angular momentum pointing in all possible directions, with an amplitude for each direction given by a spherical harmonic. Such states are eigenstates of both the total helium angular momentum (quantum number $L_{\mathrm{v}}$ ) and also its projection on the laboratory $Z$ axis (quantum number $M_{\mathrm{v}}$ ). The vortex lines we have discussed have total squared angular momentum that span the range $(N \hbar)^{2}$ to 0 as $x_{0}$ goes from 0 to $R$. Semiclassical quantization will restrict $x_{0}$ to $N-1$ values with total angular momentum quantum number equal to $L_{\mathrm{v}}=N-1, N-2, \ldots, 1$. The straight line vortex is not allowed since the length of the total angular momentum of the allowed vortex solutions is $\left|\boldsymbol{L}_{\mathrm{v}}\right|=\hbar \sqrt{L_{\mathrm{v}}\left(L_{\mathrm{v}}+1\right)}$, and the straight vortex solution has $\left|\boldsymbol{L}_{\mathrm{v}}\right|=\hbar N$. Each vortex solution with integer $L_{\mathrm{v}}$ quantum number has a $\left(2 L_{\mathrm{v}}+1\right)$-fold $M$-degeneracy.
This set of solutions gives $N^{2}$ semiclassical vortex states for a droplet of $N$ helium atoms. In principle, there are other states that involve vibrational excitation of the vortex lines ${ }^{24}$ around these Bauer-Donnelly-Vinen vortex solutions, but these will not be considered in this work.

Figure 3 shows the calculated vortex energy and angular momentum for a droplet with $N=10^{4}$ helium atoms. It is seen from this figure that the energy of the vortex drops as the vortex is moved off axis, going monotonically to zero as the vortex is "pushed out" of the droplet. By energetic considerations alone, this would imply that the vortex solutions are unstable. However, for an isolated droplet, one must also conserve angular momentum. A vortex can lower its energy by producing a ripplon if the derivative of the vortex energy with respect to total angular momentum, $\Omega^{\prime}$ (with units of angular velocity), is greater than the $E / L$ ratio of the $L=2$ ripplon. Figure 5 shows $\Omega^{\prime}$ for vortices as a function of their minimum approach distance to the $z$ axis, normalized to the energy per unit angular momentum of this ripplon mode. $\Omega^{\prime}$ was evaluated by calculation of both $E$ and $L$ for $10^{3}$ values of $x_{0}$ for each droplet size, and using $\Omega^{\prime}=\frac{d E}{d x_{0}} / \frac{d L}{d x_{0}}$, with each derivative evaluated by finite difference of the calculated points. It is evident that the vortex is stable to ripplon production (has a normalized value of $\Omega^{\prime}$ less than unity) for most of its range. Table@list the minimum energy and angular momentum of the curved vortex states that are thus stable.

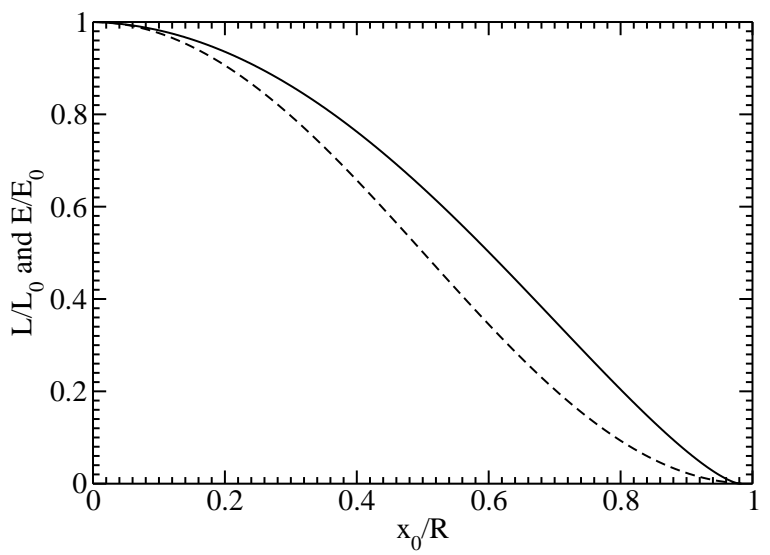

FIG. 3: Energy (solid line) and angular momentum (dashed line) of a curved vortex solution as a function of the distance of closest approach of the vortex from the $z$ axis. These results are for a $N=10^{4}$ helium atom droplet. The energy $E_{0}$ of the straight vortex is given in Expression (1); its angular momentum is $L_{0}=N \hbar$.

Because the energy and angular momentum in the hollow core model arise entirely from helium motion, we had anticipated that $\Omega^{\prime}$ would be equal to $\Omega$, the angular velocity of the curved vortex solution around the $z$ axis. For vortex rings in the local induction approximation, Rayfield and Reif 18 found that the vortex velocity satisfied $v=d E / d p$, where $p$ is the net helium linear momentum due to helium flow around the vortex ring. This insures 


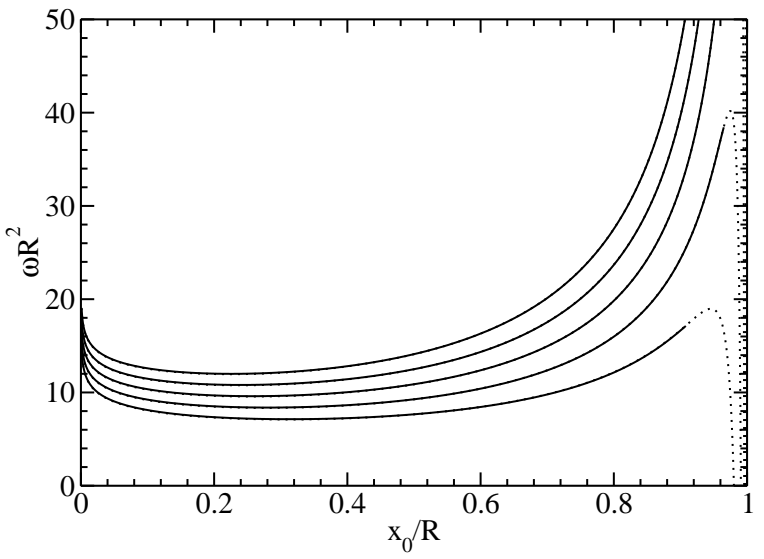

FIG. 4: Angular velocity as a function of distance of closest approach, calculated for droplets with $N=10^{2}, 10^{3}, 10^{4}, 10^{5}$, $10^{6}$ (bottom to top) He atoms. Plotted is $\omega R^{2}=4 \pi \Omega R^{2} / \kappa$, a dimensionless quantity. Solid lines are computations using the model described in Appendix B dotted lines use the model of Ref. 22. As discussed in the Appendix, the local-induction approximation breaks down as $x_{0} \rightarrow R$.

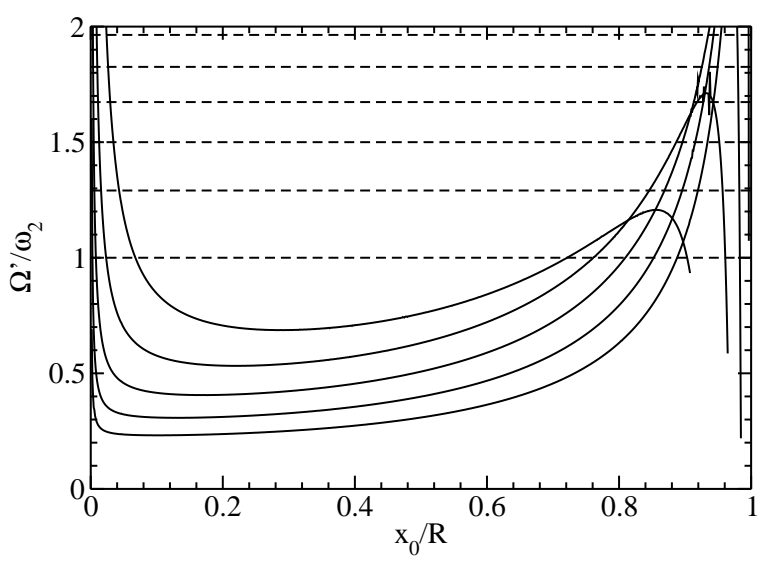

FIG. 5: Comparison of the vortex "angular velocity" $\Omega^{\prime}=$ $\mathrm{d} E / \mathrm{d} L$ and the angular velocities $\omega_{L}$ of the lowest ripplons $(L=2 \ldots 7)$, as a function of distance of closest approach to the $z$ axis, for droplets with $N=10^{2}, 10^{3}, 10^{4}, 10^{5}, 10^{6}, 10^{7}$ (top to bottom) He atoms. All angular velocities are shown as ratios to $\omega_{2}=\sqrt{8 \pi \sigma /(3 m N)}$.

that as $x_{0} \rightarrow R$ (but $R-x_{0} \gg a$ so the local induction approximation still holds), $\Omega^{\prime} \rightarrow \Omega$. For a vertical vortex in a cylinder (where $E$ and $L$ have simple analytical solutions), we have demonstrated that $\Omega^{\prime}=\Omega^{\prime}$. For our solutions of curved vortex lines in a spherical droplet, we however find that $\Omega^{\prime} \neq \Omega^{\prime}$, as demonstrated in Figure 6] where both are compared as a function of $x_{0} / R$ for various droplet sizes. It appears from our calculations that $\Omega^{\prime} \rightarrow \Omega$ as $N \rightarrow \infty$ (except for the log divergence of $\Omega$ as $x_{0} \rightarrow 0$ ), but the rate of convergence is rather slow. It remains to be established whether the discrepancy between $\Omega$ and $\Omega^{\prime}$ is due to an error in calculation or whether it is real.

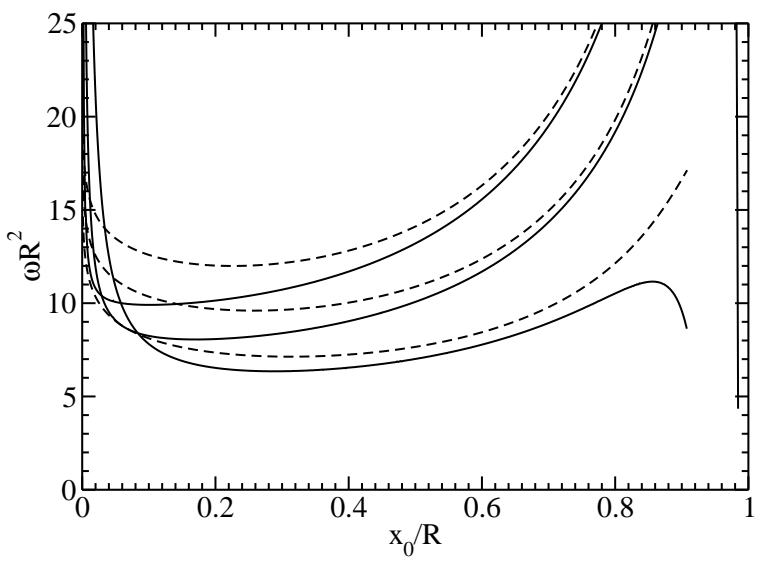

FIG. 6: Comparison of the computed reduced angular velocity $\omega=\frac{4 \pi}{\kappa} \Omega$ (dashed lines) and the quantity $\omega^{\prime}=\frac{4 \pi}{\kappa} \mathrm{d} E / \mathrm{d} L$ (solid lines), for droplets with $N=10^{2}, 10^{4}, 10^{6}$ atoms (bottom to top).

It is evident from figure 6 that $\Omega$ peaks for the straight vortex $\left(x_{0}=0\right)$; more careful analysis shows that there is in fact a logarithmic divergence: $\Omega \approx \frac{\kappa}{2 \pi R^{2}} \ln \frac{4 R^{2}}{a x_{0} e}$ as $x_{0} \rightarrow 0$. In the limit that the vortex line moves near the surface of the droplet, our solutions no longer provide meaningful estimates as the vortex ring radius $R-x_{0}$ approaches the value of the core radius $a$. In addition to the expected breakdown of the local induction approximation in this limit, such a vortex would be localized in the region of highly inhomogeneous density near the helium surface. Feynman has suggested that the limit of a vortex ring of atomic scale is the roton quasiparticle excitation 25

In order to test that there was no numerical error in the evaluation of the integral expressions for $L_{\mathrm{v}}$ and $E_{\mathrm{v}}$ reported in this work, calculations for selected values of $a / R$ were performed with totally independent routines written by the two authors, using distinct programing packages (Mathematica and Mathcad), and the values of $\Omega, L_{\mathrm{v}}$, and $E_{\mathrm{v}}$ were found to agree to six significant figures.

\section{POSSIBLE SPECTROSCOPIC SIGNATURES OF MOLECULES BOUND TO VORTICES}

It is well know in bulk liquid helium that impurities tend to bind to vortex lines or vortex rings. The binding of ions to vortex rings was the basis of the remarkable experiment of Rayfield and Reif 18 that measured many of the properties of ring vortices. Calculations by both Dalfovo et al $\stackrel{4}{*}$ and by Draeger and Ceperley ${ }^{5}$ have demonstrated that atomic or linear molecular impurities in a helium nanodroplet bind to linear, maximum angular momentum vortex lines. For technical reasons, both these calculations required cylindrical symmetry, and thus could not study the energy change upon ro- 
tation of the molecule with respect to the vortex axis. However, it is generally believed that the binding energy is maximized by aligning the linear molecular axis with the vortex since this displaces the maximum amount of helium from near the vortex core, where it has the highest kinetic energy; however, the increased helium density in the first solvation layer around the solute may in part counteract this effect. This suggests that the anisotropy in the binding energy should be of comparable magnitude as the binding itself, but this natural expectation has yet to be checked by calculations with a realistic helium density profile. It seems likely that the magnitude of the anisotropy of the binding for linear molecules, such as HCN and HCCCN, is much higher than the rotational constant of these molecules in liquid helium. If this was correct, then the gas phase-like rotational structure of these molecules would be quenched, leading to a rovibrational spectrum dominated by a $\mathrm{Q}$ branch; such $\mathrm{Q}$ branches are, however, absent in the observed spectra of these molecules. ${ }^{36}$ This suggests that at most a small fraction of the droplets probed in these experiments could have such a linear vortex line excitation. It is further noted that the vibrational spectrum of the spherical top molecule $\mathrm{SF}_{6}$ is also expected to be a sensitive probe for the presence of a vortex line, as the highly anisotropic helium density created by the vortex core would lift the triple degeneracy of IR active fundamental modes, producing a spectroscopic structure in qualitative disagreement with what has been observed ${ }^{6}$

Molecules are also expected to bind and align with curved vortex lines, as long as the solute kinetic energy required for it to move with the curved vortex line does not exceed the binding energy of the solute molecule to the stationary vortex. The velocity of a curved vortex increases smoothly from zero when $x_{0}=0$ to a maximum value (given in table 1 ) when the vortex becomes unstable to ripplon formation. For a molecule with a translational mass of $100 \mathrm{u}$, the maximum kinetic energy that the molecule requires to stay bound to a curved vortex line is between 19.1 and $1.1 \mathrm{~K}$ as $N$ varies between $10^{2}$ and $10^{6}$ (see Table $\amalg$ ). This kinetic energy can be compared to binding energies to the straight vortex of $5.0,4.4$, and $7.7 \mathrm{~K}$ for $\mathrm{Xe}, \mathrm{HCN}$, and $\mathrm{SF}_{6}$, respectively, as calculated by DFT. 4 Thus, for the least stable curved vortex lines in smaller droplets, the molecules may become unpinned. This suggests that it would be useful to have improved binding energy estimates for molecules, particularly to curved vortex states.

\section{VORTEX FORMATION MECHANISMS}

A possible explanation for the failure of existing experiments to detect droplets with vortex lines is that they cannot be formed in the first place, perhaps because of the high energy and angular momentum required. We will consider the probability of events that deposit sufficient energy and angular momentum in the droplets such that vortex formation can in principle take place.

In most experiments, the droplets grow out of a supercooled gas ${ }^{26}$ In this situation, the large droplets probably grow at least in part by coalescence of smaller droplets. If we consider the coalescence of two droplets with $\mathrm{N} / 2$ atoms that collide with an average impact parameter ( $2 / 3$ of either's diameter), the minimum relative velocity required to deposit an angular momentum of $N \hbar$ in the combined droplet is $v_{\text {rel }}=270 N^{-1 / 3} \mathrm{~m} / \mathrm{s}$, which is $58-2.7 \mathrm{~m} / \mathrm{s}$ for $N=10^{2}-10^{6}$. Experiments of Toennies et $a l^{27}$ have found that the expansions produce droplets with a final speed ratio of $\approx 100$, which implies final relative velocities of $\approx 8 \mathrm{~m} / \mathrm{s}$ for expansion from a $20 \mathrm{~K}$ source. While such relative collision velocities are, except for the largest droplets, less than those required to produce a vortex line with maximum angular momentum (straight vortex), they are sufficient to produce curved vortices. Further, the above estimates are only average values, and the relative velocities in the part of the expansion where the droplets undergo substantial growth are likely significantly higher. Experiments that produce droplets above $N \approx 5 \times 10^{4}$ typically use expansion conditions such that liquid helium is ejected through a cold nozzle into vacuum, and fragments by cavitation as the pressure falls far below the equilibrium vapor pressure. This break-up is believed to generate considerable turbulence, and thus may lead to dense vortex formation. 28.29

The "pick-up" process $\stackrel{30}{,}$ by which droplets are doped with solutes, should often deposit enough angular momentum, and almost always enough energy, to form a linear vortex line, particularly for the pickup of heavy molecules by not too large droplets. It has been predicted that vortices are nucleated when the velocity of an impurity exceeds to sound velocity in helium, 31 which is well below at least the typical impact speed of atoms or molecules striking helium nanodroplets. Consider the pickup of atoms or molecules of mass $M$ from a thermal gas at temperature $T$ by droplets with laboratory frame velocity $v_{\mathrm{d}}$. Integration over the Maxwell-Boltzmann distribution for the gas gives the probability density $P_{\mathrm{r}}$ for the relative velocity $v_{\mathrm{r}}$ between droplet and gas

$$
P_{\mathrm{r}}\left(v_{\mathrm{r}}\right)=\sqrt{\frac{2 M}{\pi k T}} \frac{v_{\mathrm{r}}}{v_{\mathrm{d}}} \sinh \left[\frac{M v_{\mathrm{r}} v_{\mathrm{d}}}{k T}\right] \exp \left[-\frac{M\left(v_{\mathrm{r}}^{2}+v_{\mathrm{d}}^{2}\right)}{2 k T}\right] .
$$

From this distribution, the average relative collisional speed, $\bar{v}_{\mathrm{r}}$, is calculated to be

$$
\bar{v}_{\mathrm{r}}=\sqrt{\frac{2 k T}{\pi M}} \exp \left[-\frac{M v_{\mathrm{d}}^{2}}{2 k T}\right]+\left(v_{\mathrm{d}}+\frac{k T}{M v_{\mathrm{d}}}\right) \operatorname{erf} \sqrt{\frac{M v_{\mathrm{d}}^{2}}{2 k T}} .
$$

For a given impact parameter $b$, all collisions with relative velocity $v_{\mathrm{r}} \geq v_{\mathrm{m}}(b)=\hbar / b \cdot(1 / m+N / M)$ result in angular momentum of at least $N \hbar$, enough to form a straight vortex. The fraction of such collisions is

$$
\Phi(b)=\frac{\int_{v_{\mathrm{m}}(b)}^{\infty} v_{\mathrm{r}} P_{\mathrm{r}}\left(v_{\mathrm{r}}\right) \mathrm{d} v_{\mathrm{r}}}{\int_{0}^{\infty} v_{\mathrm{r}} P_{\mathrm{r}}\left(v_{\mathrm{r}}\right) \mathrm{d} v_{\mathrm{r}}}
$$


If we assume that the the pickup probability is independent of collisional impact parameter $b$ for $b \leq R=$ $r_{0} N^{1 / 3}$, and falls abruptly to zero for $b>R$, then the fraction of resulting doped droplets that have at least $N \hbar$ of angular momentum from the pickup process is

$$
P_{\geq N \hbar}=\int_{0}^{R} \frac{2 \pi b \mathrm{~d} b}{\pi R^{2}} \Phi(b) .
$$

Figure 7 shows plots of the fraction of pickup collisions that result in an angular momentum greater than $N \hbar$, as a function of $N$ for a number of solutes. Figure 8 shows, for droplets of $N=10^{4}$ helium atoms, the distribution of collisional angular momenta deposited by pickup of the same solutes. In many cases relevant to previously reported experiments, the pickup process has a fair probability to deposit sufficient angular momentum to create a straight vortex, and almost always sufficient angular momentum to create curved vortex lines. Thus, the lack of observation of vortex lines does not appear to be due to a lack of initial angular momentum to form them.

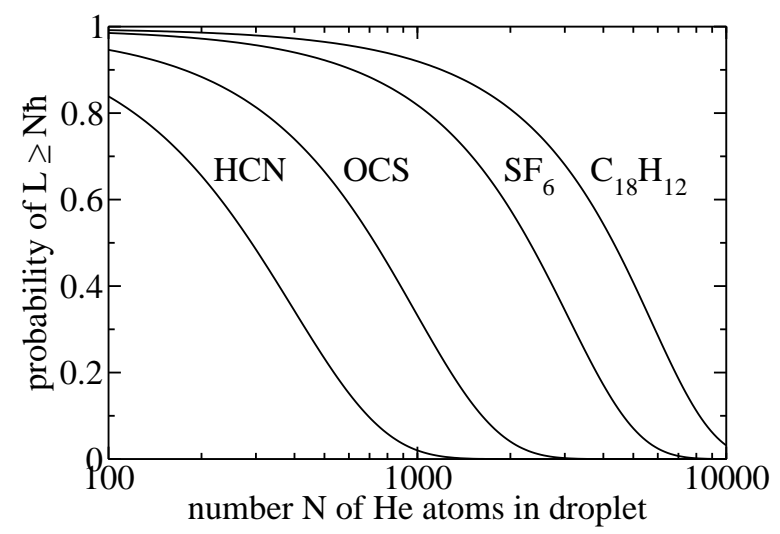

FIG. 7: Probability of a pickup collision depositing more than $N \hbar$ units of angular momentum in the droplet, as function of droplet size $N$. Droplet velocity $v_{\mathrm{d}}=456 \mathrm{~m} / \mathrm{s}$ (20 K supersonic expansion); gas temperature $T=300 \mathrm{~K}$.

\section{STABILITY ANALYSIS OF VORTEX STATES}

An alternative explanation for the lack of evidence of vortex lines in experiments to date could be that vortex lines are unstable, and decay or are expelled from the droplets in a time short compared to the time between the pickup of solutes and the spectroscopic detection of the doped droplets. In this section, several possible vortex destruction pathways are considered, concentrating on the simplest case of the linear vortex line.

A helium nanodroplet excited with a linear vortex line has an angular momentum of $N \hbar$, much higher than the magnitude of the angular momentum thermally present in the ripplons at the droplet temperature of $\approx 0.38 \mathrm{~K}$ that has been experimentally found for helium nanodroplets cooled by evaporation $\underline{\underline{6}}$ For droplets in the size

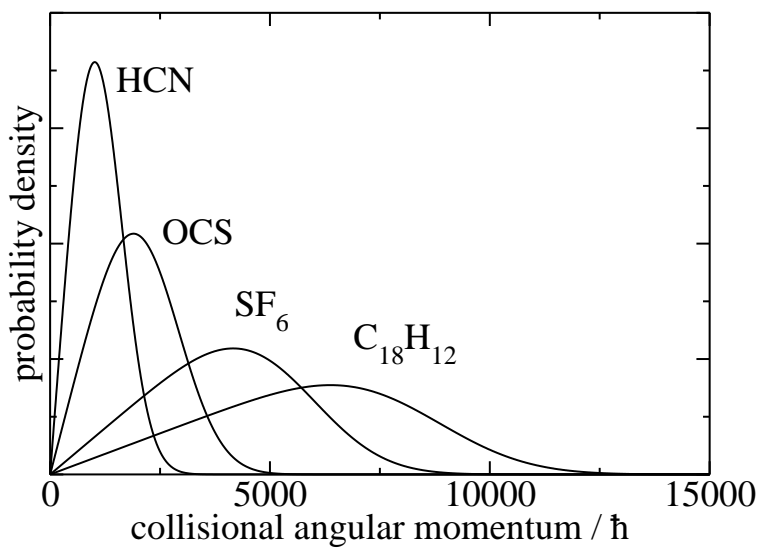

FIG. 8: Probability densities of collisional angular momenta during pickup of various molecules by a droplet with $N=10^{4}$ He atoms. Droplet velocity $v_{\mathrm{d}}=456 \mathrm{~m} / \mathrm{s}(20 \mathrm{~K}$ supersonic expansion); gas temperature $T=300 \mathrm{~K}$. For other droplet sizes, the shapes of the distributions are the same except for a correction due to changes in the reduced mass, and scaling of the average collisional angular momentum as $N^{1 / 3}$.

range of experiments reported to date, the thermal excitation of phonon states is negligible in comparison to that of the ripplons $\stackrel{20}{\underline{n}}$ The lowest frequency $(L=2)$ ripplon mode has the lowest energy per unit angular momentum of any of the helium excitations except vortices, but this value is still higher than the corresponding value for the linear vortex, as indicated in Figure 1] Table [shows the ratio of excitation energies and angular momenta for the $L=2$ ripplon and the linear vortex for a range of helium droplet sizes. While decay of the vortex state is highly exothermic, this is only true if one ignores the requirement of conservation of angular momentum; this neglect is appropriate for helium contained in a vessel with walls, to which the angular momentum can be transferred, but not for a droplet isolated in a high vacuum chamber. When angular momentum conservation is enforced, the decay of the linear vortex line into ripplons is highly endothermic and thus cannot occur for a droplet unless the droplet also contains a counter-angular momentum in ripplons that is nearly as large as the angular momentum of the vortex itself. Table【also contains the thermal average value $\sqrt{\langle L(L+1)\rangle}$ of ripplon angular momentum for different size droplets at $0.38 \mathrm{~K}{ }^{32}$ it is only a small fraction of that of the straight vortex. Exchange of angular momentum between thermally populated ripplons and a vortex could at most allow the vortex to move slightly off axis.

Helium nanodroplets can shed angular momentum by helium atom evaporation. A helium atom evaporating with momentum $p$ can carry away a maximum angular momentum of $\approx p R$, which reduces the internal energy of the droplet by $E_{\mathrm{b}}+p^{2} /\left(2 m_{\mathrm{He}}\right)$, where $E_{\mathrm{b}}$ is the binding energy of the helium atoms to the droplet. For finite droplets, $E_{\mathrm{b}}$ is reduced from the bulk value due to a term that reflects the change in surface energy upon evapora- 
tion of an atom 37 Table $\llbracket$ shows the maximum angular momentum that can be lost by single atom evaporation using the entire energy of the linear vortex line ${ }^{38}$ In every case, that angular momentum is far lower than the angular momentum of the vortex line that must be carried off for the vortex line to decay. Evaporation of multiple helium atoms will increase the angular momentum that can be carried off for the same total helium kinetic energy. Because the available kinetic energy is reduced by the increased binding energy of the multiple helium atoms, there is a number of evaporated atoms that allows the maximum angular momentum to be removed. Table 【 also lists this amount of angular momentum for a range of droplet sizes $\stackrel{39}{\underline{\underline{3}}}$ It is evident that in all cases, this maximal amount of angular momentum lost by evaporation is far below that which is needed for the vortex line to decay while conserving angular momentum.

The above analysis assumed that the helium atoms evaporated as isolated atoms. An alternative decay mechanism is fission of the droplet: in this case, the loss of helium binding energy is only due to the increase in surface energy, which is higher for the two droplets than for the original nearly-spherical droplet. Fission of a droplet into two equal-size droplets costs far more energy than that contained in a vortex line, even neglecting the required kinetic energy for relative motion of the fragments. Table \ contains the fragmentation that carries away the maximum possible quantity of angular momentum, taking the original droplet radius as the impact parameter of the departing fragments $\stackrel{40}{-}$ Yet again, this is far below the angular momentum of the linear vortex.

The above considerations show that the simultaneous constraints of conservation of energy and angular momentum prevent decay of straight vortices in a pure droplet by any of the mechanisms that are known to the authors. Adding a molecule to the droplet will further decrease the energy per unit angular momentum of the vortex as long as the molecule remains bound to the vortex. It thus appears that droplets with a vortex line should be stable to decay.

How can we understand the failure to date to observe vortex lines in helium nanodroplet isolation spectroscopy? The answer is probably related to the fact that under typical experimental conditions, the above mechanisms, by putting sufficient angular momentum into the droplets to form vortex lines, add far more than the minimum energy required to do so. The density of ripplon states grows very rapidly with increasing energy ${ }^{33}$ it may well be that at the energy and angular momentum of a droplet following coalescence or pickup, the fraction of states that contain a vortex line is a totally insignificant fraction of the total density of states, and that droplets will shed sufficient angular momentum along with energy as they evaporatively cool that they have a negligible chance to be trapped in one of the metastable solutions containing a vortex. We have recently carried out Monte Carlo cooling calculations, using statistical reaction rate theory, for the evaporative cooling of pure and doped helium nanodroplets, conserving both energy and angular momentum 34 considering only ripplon excitations of the droplets. In the future, we hope to extend that work to include the spectrum of vortex line excitations as well, which it is hoped will shed further light on the still unsolved problem of why spectra of molecules bound to vortex lines has yet to be observed in Helium Nanodroplet Isolation Spectroscopy.

\section{APPENDIX A: HOLLOW-CORE MODEL}

In the local-induction approximation, the flow field around a curved vortex is approximated locally by the flow around a vortex ring of equal radius of curvature. The velocity potential for the flow around a vortex ring in the $x-y$ plane, with radius $\mathcal{R}$, centered at the origin and moving in the $+z$ direction, can be computed from Eq. (2):

$$
\begin{array}{r}
\phi=\frac{\kappa \mathcal{R} z}{\pi\left(\mathcal{R}^{2}-r^{2}-z^{2}\right) \sqrt{(\mathcal{R}+r)^{2}+z^{2}}}\left(\left(\mathcal{R}+\sqrt{r^{2}+z^{2}}\right) \cdot \Xi\left[\frac{(\mathcal{R}-r)^{2}+z^{2}}{\mathcal{R}^{2}+r^{2}+z^{2}-2 \mathcal{R} \sqrt{r^{2}+z^{2}}}, \frac{(\mathcal{R}-r)^{2}+z^{2}}{(\mathcal{R}+r)^{2}+z^{2}}\right]\right. \\
\left.+\left(\mathcal{R}-\sqrt{r^{2}+z^{2}}\right) \cdot \Xi\left[\frac{(\mathcal{R}-r)^{2}+z^{2}}{\mathcal{R}^{2}+r^{2}+z^{2}+2 \mathcal{R} \sqrt{r^{2}+z^{2}}}, \frac{(\mathcal{R}-r)^{2}+z^{2}}{(\mathcal{R}+r)^{2}+z^{2}}\right]\right)+\frac{\kappa}{2} \operatorname{sign}(z),
\end{array}
$$

with the function

$$
\Xi[n, m]=i\left(\Pi(n \mid m)-\frac{1}{\sqrt{m}} \Pi\left(\frac{n}{m} \mid \frac{1}{m}\right)\right)
$$

in terms of complete elliptic integrals of the third kind, $\Pi(n \mid m) ; \kappa=h / m$ is the quantum of circulation. Series expansion of Eq. (A1) around the vortex singularity, with

$$
\begin{gathered}
(r, z)=\mathcal{R} \cdot(1+\zeta \cos \vartheta, \zeta \sin \vartheta) \text { and } 0<\zeta \ll 1, \text { yields } \\
\phi \approx \frac{\kappa \vartheta}{2 \pi}-\frac{\kappa \zeta}{4 \pi} \ln \frac{8}{\zeta} \sin \vartheta+\frac{3 \kappa \zeta^{2}}{32 \pi}\left(\ln \frac{8}{\zeta}-\frac{5}{6}\right) \sin 2 \vartheta .
\end{gathered}
$$

In the hollow-core model, we assume that the vortex singularity is surrounded by an empty "vortex core" of radius $a$, outside of which the helium density is constant 
and equal to the bulk value, $\rho_{\mathrm{b}}$. The helium velocity field is given by $\boldsymbol{v}=-\boldsymbol{\nabla} \phi$ : the first term in Eq. A3 is the source of the looping helium flow around the vortex, the second term gives rise to the forward motion of the vortex ring with speed

$$
u=\frac{\kappa}{4 \pi \mathcal{R}}\left[\ln \frac{8 \mathcal{R}}{a}-1\right]
$$

along the $+z$ axis, and the third term allows us to estimate the residual flow across the vortex core surface due to the fact that the exact vortex core surface is not circular in cross section: the ratio of the rms velocity of this residual normal flow to the forward velocity is $3 a /(4 \mathcal{R} \sqrt{2})$ as $a / \mathcal{R} \rightarrow 0$.

Rayfield and Reif 18 have experimentally determined the forward velocity $u$ of ring vortices, and determined a core radius of $\tilde{a}=1.00 \AA$ by using the formula $u=\frac{\kappa}{4 \pi \mathcal{R}}\left[\ln \frac{8 \mathcal{R}}{\tilde{a}}-\frac{1}{2}\right]$. Comparison of this expression to Eq. (A44) yields $a=\tilde{a} / \sqrt{e}=0.607 \AA$, which is the core radius that we use in this work for the hollow-core model.

\section{APPENDIX B: VORTEX SHAPE CALCULATION}

A vortex, $\boldsymbol{s}(\ell)$, is most generally described as a curve in the $x, z$ plane parametrized by its length $\ell$; threedimensional vortex curves are always longer and thus higher in energy for given boundary conditions. ${ }^{22}$ The local vortex curvature is

$$
\chi(\ell)=\frac{x^{\prime \prime}(\ell)}{z^{\prime}(\ell)}=-\frac{z^{\prime \prime}(\ell)}{x^{\prime}(\ell)} .
$$

In the hollow-core model with the local-induction approximation, the magnitude of the vortex velocity is determined from the core radius and the local curvature, and its direction is given by the binormal vector (the normalized cross product of $\frac{d s}{d \ell}$ and $\left.\frac{d^{2} s}{d \ell^{2}}\right)$. The magnitude of the velocity is given by Eq. (A4):

$$
v(\ell)=-\frac{\kappa}{4 \pi} \chi(\ell)\left[\ln \frac{a \chi(\ell)}{8}+1\right] .
$$

For a vortex to rotate around the $z$ axis at angular velocity $\Omega$ without changing shape, it must satisfy $v(\ell)=$ $\Omega x(\ell)$, which leads to the coupled differential equations that determine the vortex shape:

$$
\left\{x^{\prime \prime}(\ell), z^{\prime \prime}(\ell)\right\}=\frac{\omega x(\ell)}{W_{-1}[-a \omega x(\ell) e / 8]}\left\{-z^{\prime}(\ell), x^{\prime}(\ell)\right\},
$$

where we have used $\omega=4 \pi \Omega / \kappa$ and the Lambert function $W_{-1}(x)^{41}$ defined as the smaller of the two real solutions of $x=y e^{y}$ for $-1 / e \leq x<0$. Combined with the initial conditions $\{x(0), z(0)\}=\left\{x_{0}, 0\right\}$ and $\left\{x^{\prime}(0), z^{\prime}(0)\right\}=\{0,1\}$, Eq. (B33) yields the desired vortex shape functions by numerical integration. For a given minimum approach distance $x_{0}$ to the $z$ axis, we must pick the parameter $\omega$, and thus the angular velocity $\Omega$, such that the resulting vortex line intersects the droplet surface perpendicularly; for a spherical droplet with radius $R$, this condition is $x\left(\ell_{1}\right) z^{\prime}\left(\ell_{1}\right)=z\left(\ell_{1}\right) x^{\prime}\left(\ell_{1}\right)$ with $x\left(\ell_{1}\right)^{2}+z\left(\ell_{1}\right)^{2}=R^{2}$.

This procedure differs from the one given in Ref. 22 in that Bauer et al. neglect the variation of the Lambert function, and replace the denominator of Eq. B3 by a constant. For droplets consisting of $100 \leq N \leq 100000$ helium atoms, the error of this approximation is below $0.6 \%$ for the evaluation of the angular velocity.

Figure 4 shows angular velocities of curved vortices computed with both models.

As $x_{0} \rightarrow R$, the vortex solutions are close to half-circles with radius $R-x_{0}$. We expect the hollow-core model to break down if $R-x_{0} \lesssim a$. A clear sign of this breakdown is that for $x_{0} \rightarrow R$, the Lambert function in Eq. (B33) starts giving complex values as the droplet surface is approached $(a \omega x(\ell) e / 8>1 / e)$. In the simplified model of Ref. 22, the angular velocity turns toward negative values for $R-x_{0}<a$, invalidating that model as well. It is to be noted that in this limit, the description of the vortex core as a cylindrical tube around the vortex is very inaccurate.

\section{APPENDIX C: CORE CORRECTIONS}

Bauer et al. show 22 that the angular momentum and kinetic energy of a droplet with vortex can be written as surface integrals:

$$
\begin{aligned}
& \boldsymbol{L}_{\mathrm{v}}=m \rho_{\mathrm{b}} \int_{\Sigma} \phi \boldsymbol{r} \times \mathrm{d} \boldsymbol{S} \\
& E_{\mathrm{v}}=\frac{1}{2} m \rho_{\mathrm{b}} \int_{\Sigma} \phi \boldsymbol{v} \cdot \mathrm{d} \boldsymbol{S},
\end{aligned}
$$

where $\Sigma$ is the surface of a connected volume of helium with no branch cuts in the velocity potential $\phi$, and $\boldsymbol{v}=$ $-\nabla \phi$. While most of the angular momentum and kinetic energy comes from the surface integrations of Eqs. (516), there are corrections due to the surface integrations of Eqs. (C1C2) over the vortex core. Using Eqs. (A3), (B1), and (B3), we find these corrections to be

$$
\begin{aligned}
\delta L_{\mathrm{v}, z} & \approx \frac{m \rho \kappa}{a \omega^{2}}\left[C_{1,0}-\frac{1}{4} C_{2,0}+\frac{1}{2} C_{2,1}\right] \\
\delta E_{\mathrm{v}} & \approx \frac{m \rho \kappa^{2}}{8 \pi a \omega}\left[-C_{1,0}+\frac{1}{4} C_{2,0}-\frac{1}{8} C_{2,1}\right],
\end{aligned}
$$

with

$$
C_{n, m}=(a \omega)^{n+1} \int_{\text {vortex }} \frac{x(\ell)^{n}}{W_{-1}^{m}(-a \omega x(\ell) e / 8)} \mathrm{d} \ell .
$$

From symmetry considerations, we must have $L_{\mathrm{v}, z}\left(-x_{0}\right)=L_{\mathrm{v}, z}\left(x_{0}\right)$ and $E_{\mathrm{v}}\left(-x_{0}\right)=E_{\mathrm{v}}\left(x_{0}\right)$. In fact, the first core correction $\left(C_{1,0}\right)$ adjusts $L_{\mathrm{v}, z}$ to satisfy this symmetry constraint, i.e., $L_{\mathrm{v}, z}\left(x_{0}\right)=N \hbar-\mathcal{O}\left(x_{0}^{2}\right)$. 
However, this symmetry is not exactly satisfied for $E_{\mathrm{v}}\left(x_{0}\right)$, which we assume is due to inaccuracies of the local-induction approximation.

\section{ACKNOWLEDGMENTS}

The authors would like to thank Manuel Barranco for helpful comments upon an draft of this paper. This work was supported by a grant from the National Science Foundation.
* Electronic address: lehmann@princeton.edu

1 R. J. Donnelly, Quantized Vortices in Helium II (Cambridge University Press, Cambridge, UK, 1991).

2 J. D. Close, F. Federmann, K. Hoffmann, and N. Quaas, J. Low Temp. Phys. 111, 661 (1998).

3 S. Goyal, D. L. Schutt, and G. Scoles, Phys. Rev. Lett. 69, 933 (1992).

${ }^{4}$ F. Dalfovo, R. Mayol, M. Pi, and M. Barranco, Phys. Rev. Lett. 85, 1028 (2000).

5 E. Draeger, Ph.D. thesis, University of Illinois at UrbanaChampaign (2001).

6 M. Hartmann, R. E. Miller, J. P. Toennies, and A. Vilesov, Phys. Rev. Lett. 75, 1566 (1995).

7 C. Callegari, K. K. Lehmann, R. Schmied, and G. Scoles, J. Chem. Phys. 115, 10090 (2001).

8 M. Pi, R. Mayol, M. Barranco, and F. Dalfovo, J. Low Temp. Phys. 121, 423 (2000).

9 R. Mayol, M. Pi, M. Barranco, and F. Dalfovo, Phys. Rev. Lett. 87, 145301 (2001).

10 J. Lekner, J. Phys. - Cond. Mat. 12, 4327 (2000).

11 M. Barranco, R. Mayol, M. Pi, and F. Dalfovo, J. Low Temp. Phys. 126, 281 (2002).

12 G. V. Chester, R. Metz, and L. Reatto, Phys. Rev. 175, 275 (1968).

13 F. Dalfovo, Phys. Rev. B 46, 5482 (1992).

14 G. Ortiz and D. M. Ceperley, Phys. Rev. Lett. 75, 4642 (1995).

15 S. A. Vitiello, L. Reatto, G. V. Chester, and M. H. Kalos, Phys. Rev. B 54, 1205 (1996).

16 M. Sadd, G. V. Chester, and L. Reatto, Phys. Rev. Lett. 79, 2490 (1997).

17 S. Giorgini, J. Boronat, and J. Casulleras, Phys. Rev. Lett. 77, 2754 (1996).

18 G. W. Rayfield and F. Reif, Phys. Rev. A 136, A1194 (1964).

19 J. Harms, J. P. Toennies, and F. Dalfovo, Phys. Rev. B 58, 3341 (1998).

20 D. M. Brink and S. Stringari, Z. Phys. D 15, 257 (1990).

21 G. Deville, P. Roche, N. J. Appleyard, and F. I. B. Williams, Czechoslovak J. Phys. 46, 89 (1996).

22 G. H. Bauer, R. J. Donnelly, and W. F. Vinen, J. Low Temp. Phys. 98, 47 (1995).

${ }^{23}$ C. M. Muirhead, W. F. Vinen, and R. J. Donnelly, Phil.
Trans. R. Soc. London A 311, 433 (1984).

24 L. Kiknadze and Y. Mamaladze, J. Low Temp. Phys. 127, 271 (2002).

25 R. P. Feynman, Statistical Mechanics: A Set of Lectures (Addison-Wesley, Reading, Massachusetts, 1990).

26 J. A. Northby, J. Chem. Phys. 115, 10065 (2001).

27 H. Buchenau, E. L. Knuth, J. Northby, J. P. Toennies, and C. Winkler, J. Chem. Phys. 92, 6875 (1990).

28 M. H. Zurek, Nature 317, 505 (1985).

29 M. E. Dodd, P. C. Hendry, N. S. Lawson, P. V. E. McClintock, and C. D. H. Williams, J. Low Temp. Phys. 115, 89 (1999).

30 T. E. Gough, M. Mengel, P. A. Rowntree, and G. Scoles, J. Chem. Phys. 83, 4958 (1985).

31 N. G. Berloff and P. H. Roberts, Phys. Lett. A 274, 69 (2000)

32 K. K. Lehmann, Mol. Phys. 97, 645 (1999).

33 K. K. Lehmann (2003), to be published in J. Chem. Phys.

${ }^{34}$ K. K. Lehmann and A. Dokter (2003), unpublished.

${ }^{35}$ F. Chapeau-Blondeau and A. Monir, IEEE Trans. Sig. Process. 50, 2160 (2002).

36 There have been weak Q branches observed in the spectra of polymers of these species, but these have recently been assigned to thermal excitations.

37 By differentiation of the sum of bulk and surface energies with respect to $\mathrm{He}$ particle number, one finds that $E_{\mathrm{b}}(N)=E_{\mathrm{b}}($ bulk $)-\frac{8 \pi}{3} \sigma r_{0}^{2} N^{-1 / 3}$.

38 This angular momentum is given by $\sqrt{2 m_{\mathrm{He}}\left(E_{\mathrm{HC}}(N)-E_{\mathrm{b}}(N)\right)} \cdot R(N)$.

39 This maximum angular momentum is the maximum of $\sqrt{2 m_{\mathrm{He}}\left(n E_{\mathrm{HC}}(N)-n^{2} E_{\mathrm{b}}(N)\right)} \cdot R(N)$ with respect to $n$, the number of evaporated helium atoms.

40 This angular momentum is the maximum with respect to the number of atoms in one fragment, $n$, of $\sqrt{2 \mu(n)\left(E_{\mathrm{HC}}(N)-\Delta S(n, N) \sigma\right)} \cdot R(N)$ where $\mu(n)=$ $m_{\mathrm{He}} n(N-n) / N$ is the reduced mass of the departing fragments and $\Delta S=4 \pi r_{0}^{2}\left(n^{2 / 3}+(N-n)^{2 / 3}-N^{2 / 3}\right)$ is the increase in surface area.

41 In Maple, $W_{k}(x)$ is entered as LambertW( $\left.\mathrm{k}, \mathrm{x}\right)$; in Mathematica, ProductLog $[\mathrm{k}, \mathrm{x}]$. An efficient numerical routine for the evaluation of the Lambert function is given in Ref. 35. 